\title{
Brain Computer Interface to Answer Yes-No Questions
}

\author{
Avid Roman-Gonzalez ${ }^{12}$, Natalia I. Vargas-Cuentas ${ }^{1}$, Miguel Hoyos ${ }^{1}$, Joel Diaz ${ }^{2}$, Mirko Zimic ${ }^{1 *}$ \\ *mirko.zimic@upch.pe
}

\begin{abstract}
The aim of this work is to design, implement and validate a primary communication system based on a brain-computer interface. There are people who for various reasons - are affected in their ability to externalize their communication, however, they receive and process information from different sources. This system would allow basic communication - allowing the user to answer closed questions - through thought. The system was implemented by analyzing and interpreting electrical signals from brain activity, collected through electrodes attached to the scalp. The analog electrical signals were received by a data acquisition system and digitized for computer analysis. We implemented different signal processing techniques, pattern analysis, and classification and discrimination methods. By analyzing these signals and interpreting the electrical patterns, was achieved understand answers to simple questions. The system has been validated testing with healthy volunteers in the laboratory, obtaining good results.
\end{abstract}

Keywords- BCI, EEG, brain-computer interface, communication system, yes-no questions.

\section{INTRODUCTIÓN}

$\mathrm{T}$ HE functions of speech and language have essential importance for humans, both in social relations and intellectual development. When these functions are altered as a result of brain disease, the consequences exceed in severity, other dysfunctions including blindness, deafness, and paralysis.

From a broader perspective, language is the mirror of all higher mental activity. Particularly, in the case of patients, speech and language are the vehicles through which patients report their symptoms and medical problems while the means to carry out all their interpersonal relationships [1].

Although speech and language are closely interrelated functions, they are not equivalent. A disorder of language function always reflects an abnormality of the brain, and more specifically, the dominant cerebral hemisphere. A speech condition can have a similar origin, but not in all cases; it can also be caused by certain anomalies in various parts of the brain or extra-brain mechanisms.

Language function encompasses the understanding, development and transmission of ideas and feelings through the use of verbal symbols, sounds and gestures adopted and their sequential order by the accepted rules of grammar. The term speaks, on the other hand, refers more to the articulatory and phonetic aspects of verbal expression. [1]
There are people who - for various reasons - are affected in their ability to externalize their communication. One reason for this situation is the locked-in syndrome and aphasia. Aphasia is a language disorder as a result of secondary dysfunction of specific brain areas, which are manifested in the form of changes in the spoken or written expression, or a failure to understand the language heard or read.

Currently, cases of aphasia or locked-in syndrome end up being people without any capacity for physician-patient interaction for monitoring neurological health. This situation is due to the absence of a communication system allowing interaction with these patients. Additionally, in remote villages it has a limited presence of specialized medical personnel in neurology, which further complicates the evolution of the health of these patients.

In recent years, electronics and communications technologies have evolved significantly. Today, it is possible to collect, amplify and analyze electroencephalographic (EEG) signals, to interpret specific stimuli and simple questions. This basic level of communication patient-doctor can have enormous importance for the brain health of these patients.

It has been shown that the human has a leading position in the animal world due to two faculties:

1) The ability to develop and employ verbal symbols like words and written, for communicating to other members of the species.

2) The remarkable ease to use their hands. [1]

The recording of the brain electrical activity (electroencephalogram - EEG) of the motor area is far from being a simple epiphenomenon of neuronal activity. This recording is the core essence of complex brain function.

The potentials of individual neurons vary in sync, and they control the information transfer, generating different types of rhythms. These rhythms include some cortical rhythms that are in the same frequency range of the occipital alpha rhythms $(8-13 \mathrm{~Hz})$ distributed mainly in the somatosensory cortex, known as "rhythms Mu".

The rhythm Mu is shown when the subject is at rest and blocked with the movement of the contralateral limbs or extremities [2]. This particular situation allows us to propose an opportunity to use the pattern of this rhythm that maintains a constant when one registers the activity corresponding to the upper extremity, and through algorithms, generate a type of communication using alternative brain circuits. 
The use of EEG in such applications has been shown earlier [3], demonstrating its reliability and is reinforced because it is not invasive. This kind of applications makes an impact on the quality of life of patients, especially in initial disease state where they are unable to express their thoughts, generating situations of extreme anxiety.

For several years, it has been researching and developing technology to capture electroencephalographic (EEG) signals, process and interpret them to implement a braincomputer interface, or BCI (Brain - Computer Interface). In the first years when these works were developed, they were first designed to provide an interaction channel for handicapped people who for various reasons totally lost motor skills. This type of work can be found for example in [3] where the author develops a method for interpreting EEG signals and move a cursor on a computer screen. In research [4], [5] and [6] the recent developments related to the development of BCI, the pre-processing, processing, feature extraction methods, classification, and interpretation procedures are presented.

Over the past few years, the BCI were not only applied to people with motor disabilities; but also were aimed at people with intact motor abilities for different applications. These applications could be games, applications in robotics and aerospace missions among others [5] and [7].

Recently, research works related to BCI are moving towards applications in communication with people in a vegetative state, or people who are in a coma. These studies could be found in recent work [8], [9] and [10] where the authors show the first advances in this type of application.

Currently, there are several procedures and methods for implementing the BCIs, as described in works [5], [11], [12] and [13]. The first stage is a process for understanding the EEG signals, which is a record of the electrical potentials generated by the brain and obtained by electrodes placed on the scalp surface with the help of conductive pastes and gels. The EEG signals have different rhythms in the frequency domain ([3] [12] [14]) with the following characteristics:

Rhythm Alfa or Mu: Low voltage (20-60 microvolts / 3$4 \mathrm{~mm})$ with variable morphology. High frequency $(8-13 \mathrm{~Hz})$. Rhythm Beta: Low voltage (10-15 microvolts / 1-1.5 mm) with variable morphology. High frequency $(13-25 \mathrm{~Hz}$ or + ). Theta rhythm: High Voltage (50 microvolts / 7mm). Frequency $(4-8 \mathrm{~Hz})$.

Delta Rhythm: High Voltage (70 -100 microvolts / 9 -14 $\mathrm{mm}$ ) with variable morphology. Low frequency $(4$ or $-\mathrm{Hz}$ ).

For the exact location of the electrodes on the scalp, one can use the International Positioning System 10/20 which indicates the different positions to place the sensors, depending on the area and the activity to register. Figure 1 shows these locations.

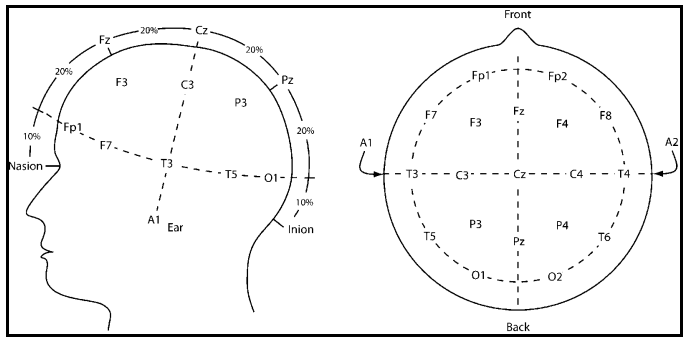

Fig. 1 International Positioning System 10/20

\section{METHODOLOGY}

This work seeks to develop a system to assist patients with neurological damage such as those described above, as works presented in [15] and [16].

In the present study, we develop a brain-computer interface, which is connected to a low-cost laptop located at the patient's bedside. The interface consists of 4 electrodes fixed in a cap which will be placed on the patient's head ensuring that the electrodes make contact with the scalp in the right positions.

The EEG signals collected by electrodes of the BCI interface will be captured and amplified by a system of analog-digital conversion to then by an ad-hoc program transmitted to the laptop.

A personal ad-hoc individualized recorded a battery of questions for each patient which is expected answers like yes/no. Before starting the process of questions, a brief and clear explanation will be transmitted to the patient to know what to do if the answer is a 'yes' and what to do if the answer is 'no'. Basically, if the patient wants to answer 'yes', he must imagine a right-hand motion; if the answer is 'no', the patient must imagine a left-hand motion. The EEG signals collected from both hemispheres of the brain may be processed to detect the patient intention.

After made some questions, the BCI interface will capture patient's EEG signals during a time window (nine seconds). These EEG signals are transmitted from the $\mathrm{BCI}$ interface to the laptop. This process is repeated until the total of questions. This information will be treated immediately by a pattern recognition algorithm that is developed in this study. Thus the collected EEG signals in response to each question will be analyzed to identify yes/no answer. Since the answers to each question will be known in advance by the system, a full report on the percentage of matches between the responses interpreted from captured EEG patterns and real answers known in advance is generated. If the patient whether or not respond randomly (probably due to a compromised neurological status), we would expect $50 \%$ of matches all issues simply by chance. Instead, by detecting a match rate significantly higher than $50 \%$, it is an indication of some degree of healthy neurological activity which is expected to occur during a favorable outcome in patients with aphasia or locked-in syndrome.

Importantly, also, the same system can be used by the treating physicians to interact in a basic way with bedridden 
patients with this syndrome. The doctor may ask questions to the patient and collect the EEG signals in response to the question. To the extent that the expected answer is 'yes' or 'no', and having explained the procedure to imagine righthand or left-hand motion if the answer is 'yes' or 'no', respectively; the EEG signals can be interpreted in real time by the doctor knowing the patient's response. In these cases the doctor may ask important questions for some time, such as: Do you have pain? Do you have a cold? Do you have any discomfort?

To demonstrate the proof of concept of how this integrated system could contribute in a meaningful way in the process of recovery of patients who suffer strokes and have lockedin syndrome and/or expressive aphasia. This system would be a highly cost-effective tool that could help bedridden patients in remote areas where there are not neurologists.

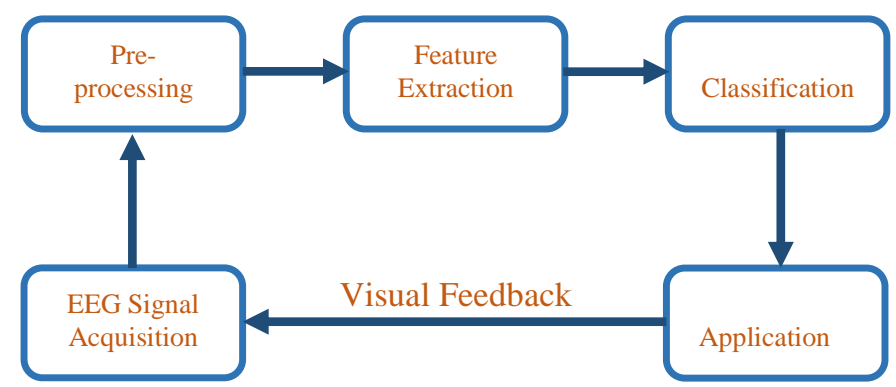

Fig. 2 Block diagram for a general BCI

System validation will be done by estimating the correlation between real thinking and system interpretation from electroencephalographic signals picked up against questions whose answers are yes/no.

For the characterization of the EEG signal, it is crucial to analyze the EEG signal in the frequency domain, precisely the $\mathrm{Mu}$ rhythm, which corresponds to the band between 8 and $13 \mathrm{~Hz}$. This rhythm is picked up in the sensorimotor zone located in the central region of the scalp. This rhythm has the peculiarity of presenting an attenuation in its amplitude when some motion is carried out, or what is more important when one intends to make some movement, or only imagining extremities motions. It is precisely this type of behaviour that we must detect through a frequency analysis of amplitude of the Fast Fourier Transform.

The classification stage is the final task of the entire process. For the entry to the classification or discrimination algorithm, we have the set of extracted features in the previous stage, and the output is an interpretation of the brain activity developed by the user.

\section{RESULTS}

For the proof of concept, the system was tested in several laboratory volunteers with normal motor capacity, and in a patient with amyotrophic lateral sclerosis (ALS).
As a result, we show the calculated error at each time instant during the 9 seconds.

Figure 3 shows the results of one tests performed on a volunteer with intact motor skills. We can observe that the error decreases and reaches its minimum point of $6.25 \%$ in the sixth second approximately.

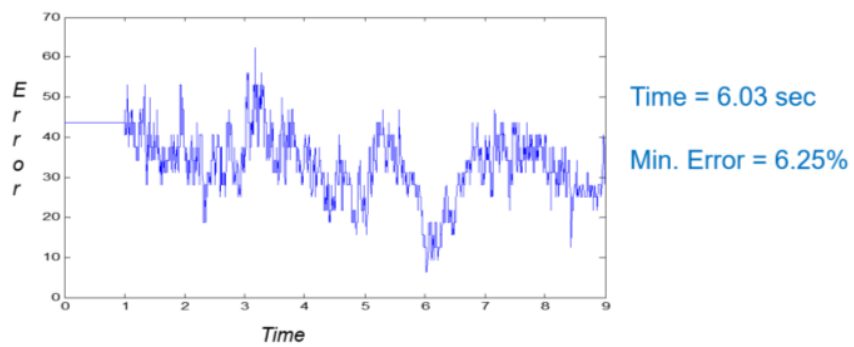

Fig. 3 Classification error of a healthy subject

In Figure 4 we can see the separation of the signals representing 'Yes' answers from 'No' answers. Clearly, a difference between the two intentions can be seen.

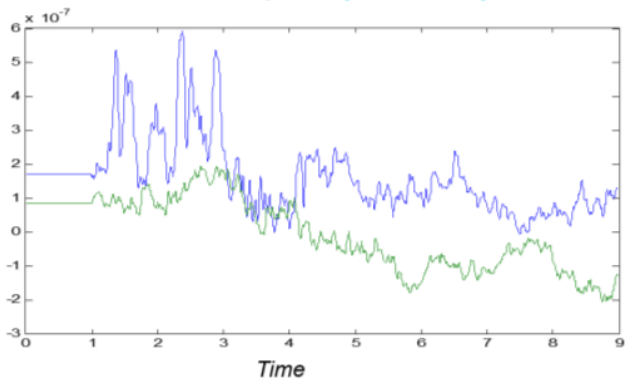

Blue $\rightarrow$ No

Green $\rightarrow$ Yes

Fig. 4 Spectral density of both signals: 'yes' and 'no'

In Figure 5, we can see the result of the classification method separating of the signals representing 'Yes' answers from 'No' answers. Classification method can separate both intentions since the fourth second.

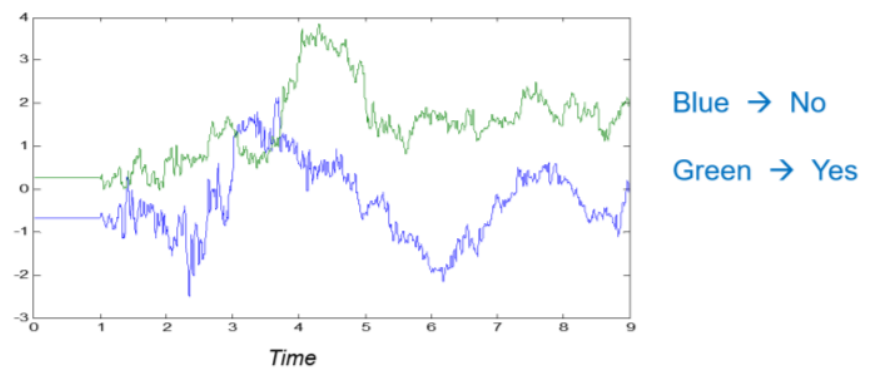

Fig. 5 Result of the classification method

In Figures 6, 7, and 8 we can observe the same results for a patient with amyotrophic lateral sclerosis.

In Figure 9, we can see a photograph of the system process tested with a laboratory volunteer with regular motor capacity. 


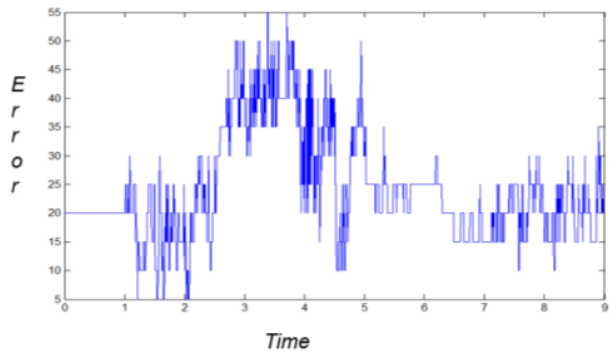

Time $=7.57 \mathrm{sec}$

Min. Error $=10 \%$

Fig. 6 Classification error of an ALS patient

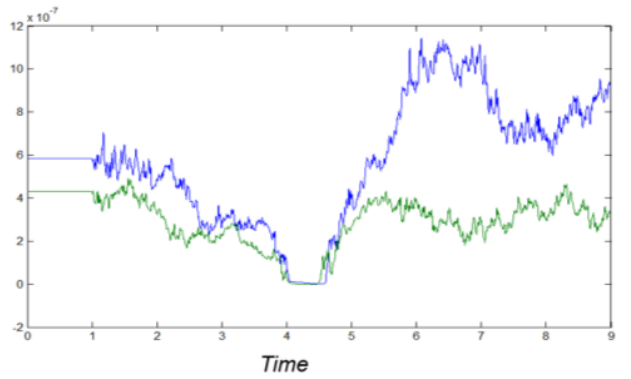

Blue $\rightarrow$ No

Green $\rightarrow$ Yes

Fig. 7 Spectral density of both signals: 'yes' and 'no'

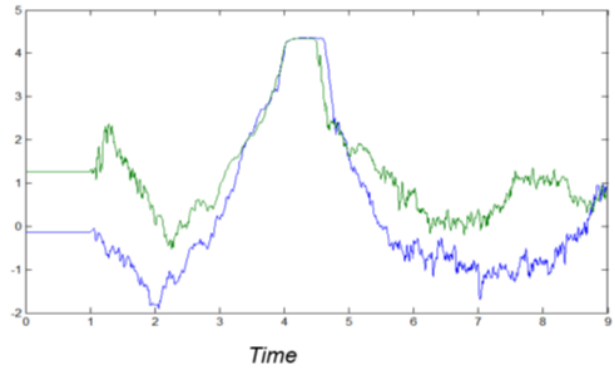

Blue $\rightarrow$ No

Green $\rightarrow$ Yes

Fig. 8 Result of the classification method

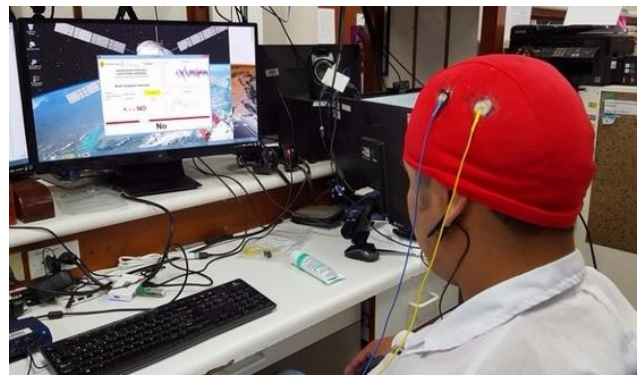

Fig. 9 Testing the system

\section{DISCUSSION}

The system was tested with good results in people with normal motor capacity; the users could dominate the use of the system quickly. However, when testing with a patient with ALS, to dominate the system was more difficult, several tests were performed over several months until an acceptable discrimination between 'yes' or 'no' answer. In spite of this, the patient did not achieve a sustained management of the system, in moments, he came to have good control, but then the error increased. This problem could be due to the mental fatigue generated by the use of the system. The errors obtained of $6.25 \%$ for people with standard motor capacity, and $10 \%$ for the patient with ALS, represent acceptable values, so, the proposal can be used as a method for essential communication.

\section{Acknowledgements}

This work was supported by FONDECYT - '01-2013Fondecyt Financiamiento de Subvención para Investigación Postdoctoral'.

\section{REFERENCES}

[1] G. Pfurtscheller, F. H. Lopes da Silva, "Event-related EEG/MEG Synchronization and Desynchronization: Basic Principles", Clinical Neurophysiology 110 (1999) 1842-1857, ELSEIVER.

[2] A. Kachenoura, L. Albera, L. Senhadji, P. Comon, "ICA: A Potential Tool for BCI System", IEEE Signal Processing Magazine 2008, 25(1): 57-88.

[3] A. Roman Gonzalez, "System of Communication and Control based on the Thought", IEEE International Conference on Human System Interaction - I'10, Poland, May. 2010, pp. 275-280.

[4] Tomita, Yohei, and Yasue Mitsukura. "Hemodynamic characteristics for improvement of EEG-BCI performance." The 6th IEEE International Conference on Human System Interaction (HSI), 2013.

[5] U. Hoffmann, J. Vesin, T. Ebrahimi, "Recent Advances in BrainComputer Interfaces", Ecole Polytechnique Federale de Laussanne (EPFL), Switzerland.

[6] Gao, Shangkai, and Xiaorong Gao. "The design and implementation of visual braincomputer interfaces." IEEE International Winter Workshop on Brain-Computer Interface (BCI), 2014

[7] Z. Ma, R. Millar, R. Hiromoto, A. Krings, "Logics in Animal Cognition: Are They Important to Brain Computer Interfaces (BCI) And Aerospace Mission", IEEE AC, 2010.

[8] T. A. Bekinschtein, F. F. Manes, M. Villareal, A. M. Owon, V. Della-Maggiore. "Functional Imaging Reveals Movement Preparatory Activity in the Vegetative State", Frontiers in Human Neuroscience, January 2011.

[9] D. Cruse, S. Chennu, C. Chatelle, T. A. Bekinschtein, D. Fernandez-Espejo, J. D. Pickard, S. Laureys, A. M. Owen. "Bedside Detection of Awareness in the Vegetative State: A Cohort Study", Thelanet, November 2011, vol. 5, Article 5.

[10] Genari, Celso Monteiro, et al. "Perception of BCI assistive technology by post-ischemic stroke patients." IEEE Biosignals and Biorobotics Conference (BRC), 2013.

[11] C. Lecocq, F. Cabestaing, "Les Interfaces Cerveau-Machine pour la Palliation du Handicap Motor Severe", LAGIS - Laboratoire d'Automatique, Génie Informatique \& Signal, Université des Sciences et Technologie de Lille, 2008.

[12] J. R. Wolpaw, N. Birbaumer, D. J. McFarland, G. Pfurtscheller, T. M. Vaughan, "Brain-Computer Interfaces for Communication and Control”, Clinical Neurophysiology 113 (2002) - ELSEIVER, pp. 767-791.

[13] J. C. Lee, D. S. Tan, "Using a Low-Cost Electroencephalograph for Task Classification in HCI Research", UIST 2006, Montreux Switzerland.

[14] M. Kirby, "Some Mathematical Ideas for Attacking the Brain Computer Interface Problem", Departement of Mathematics, Colorado State University.

[15] Hill, N. Jeremy, et al. "A practical, intuitive brain-computer interface for communicating 'yes' or 'no'by listening." Journal of neural engineering 11.3 (2014): 035003.

[16] Roman-Gonzalez, Avid. "EEG Signal Processing for BCI Applications." Human-Computer Systems Interaction: Backgrounds and Applications 2. Springer Berlin Heidelberg, 2012. 571-591. 